\title{
Systemic Lupus Erythematosus and Right Leg Weakness
}

\author{
J.L. Steckley, M.C. Tartaglia, H. Reddy, S. Huang, R. Hammond, C. Hyson
}

Can. J. Neurol. Sci. 2009; 36: 98-101

\section{Clinical Presentation - Dr. Steckley}

A 40-year old female presented to the emergency room at London Health Sciences Centre with a one week history of gradually progressive right leg weakness which had caused several falls. She also described numbness of the right arm and leg. In the two weeks leading up to admission she experienced gradually worsening shortness of breath, fatigue, anorexia, confusion, night sweats and somnolence. She also developed a herpetic rash of the right upper thigh which was treated with acyclovir and gabapentin.

Her past medical history was significant for a diagnosis of systemic lupus erythematosis (SLE) for seven years, treated at the time of admission with azathioprine $50 \mathrm{mg}$ TID (several years duration), prednisone $5 \mathrm{mg} \mathrm{BID}$, and hydroxyquine 400 mg daily. She also had a history of asthma and occasionally required Ventolin treatment. She had a 25 pack-year smoking history.

On initial examination in the ER, she was afebrile with a regular heart rate of 103 beats/minute (BPM), a blood pressure of $102 / 72$ and a low $\mathrm{O}_{2}$ saturation at $89 \%$ on room air. She was alert and oriented, with right leg weakness documented. She was admitted to the General Medicine Service for further investigation of her shortness of breath. Chest $\mathrm{x}$-rays and a ventilation-perfusion scan were unremarkable. A CT scan of the head was done and reported as abnormal. Subsequently, the neurology service was consulted.

When assessed by neurology the following day, her temperature was $38.4^{\circ} \mathrm{C}$. She looked unwell and diaphoretic. Blood pressure was 104/64 and heart rate was elevated at 115 BPM. She continued to require oxygen via nasal prongs. General examination revealed a supple neck, no bruits, and unremarkable breath and heart sounds. There were no stigmata of endocarditis. Several proximal interphalangeal joints were erythematous and swollen bilaterally.

She was drowsy and not fully oriented. She displayed poor attention and had difficulty complying with the neurological examination. Cranial nerve examination was unremarkable. A left pronater drift was demonstrated with left upper extremity hyper-reflexia. In the right leg, there was mild pyramidal pattern weakness. Her sensory exam was unremarkable to light touch and pinprick and there was no limb ataxia.

\section{Case Discussion - Dr. Tartaglia}

To summarize the case, this is a 40 -year-old woman with a subacute onset of right leg weakness and mental status changes. Her past medical history is significant for lupus for which she was immunosuppressed. In addition, she had recently developed right leg zoster and was suffering from fatigue, night sweats and shortness of breath for the last two weeks. On admission she was febrile, short of breath and required oxygen for adequate saturation. Her physical exam revealed left arm pronator drift and spasticity as well as the right leg weakness.

Thus we have multifocal disease involving mentation as well as bilateral limb involvement with some UMN signs. In addition, the patient presents with signs of systemic disease, that is, SOB, fever and night sweats. The most likely localization for her neurological signs and symptoms is the brain, although brain and spinal cord is a possibility. The differential in such a patient can be divided into three broad categories: first one can think of the problems related to the lupus directly, secondly the complications of lupus and thirdly problems related to the treatment of lupus.

Lupus is an autoimmune inflammatory disease and as such the central and peripheral nervous system is not infrequently injured in autoimmune/inflammatory diseases. The differential diagnosis necessarily includes ischemic or hemorrhagic stroke. The etiology of stroke in SLE can be thrombotic disease, secondary to cardiogenic embolisation from pericarditis or valvular disease. Accelerated atherosclerosis has been recognized in SLE as well. A high incidence of antiphospholipid antibodies places these patients in a prothrombotic state. Venous thrombosis can also cause multifocal pathology and is more common in SLE. Cerebral vasculitis can be associated with ischemic stroke or hemorrhage. Finally, immunologic disorders may lead to thrombocytopenia and multifocal intra- or extraaxial hemorrhage.

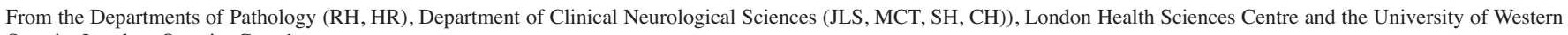
Ontario, London, Ontario, Canada.

Received June 26, 2008. Final Revisions Submitted July 8, 2008

Reprint requests to: JL Steckley, Department of Clinical Neurological Sciences, LHSC-UH, 339 Windermere Road, London, Ontario, N6A 5A5, Canada. 
Intraparenchymal inflammatory processes can also lead to multifocal lesions in the form of demyelination and/or cerebritis, both of which can be complications of SLE.

Infectious causes are an important consideration not just because of this patient's, immunocompromised state, but also because it may be the pathology most amenable to treatment. Patients with SLE may suffer immunocompromise as a consequence of their disease (ie. leukopenia) and as a secondary consequence of treatment. Although no signs of meningeal irritation were noted, a meningitis or meningoencephalitis should be considered and cerebrospinal fluid (CSF) sampling is warranted. Multifocal abscesses could also account for this presentation. Given her immunocompromised state, suspicion of opportunistic viruses (JC virus, Herpes viruses, etc.), bacteria (Listeria, Mycobacteria, etc.), fungi (Candida, Aspergillus, etc.) and protozoa (toxoplasma, etc.) would be warranted.

Another complication of chronic immune suppression and a possible explanation for this patient's multifocal presentation is primary central nervous system (CNS) lymphoma. Of course, one cannot exclude any other CNS tumors, whether primary or metastatic.

\section{DR. STECKLEY}

Initial bloodwork values were reported as follows: $\mathrm{Na}^{+} 128$ $\mathrm{mmol} / \mathrm{L}, \mathrm{K}^{+} 3.7 \mathrm{mmol} / \mathrm{L}$, urea $25.9 \mathrm{mmol} / \mathrm{L}$, creatinine 280 $\mathrm{mmol} / \mathrm{L}$, INR 1.2 , PTT normal, WBC $15.3 \times 10^{9} / \mathrm{L}$ with neutrophilia at $13.5 \times 10^{9} / \mathrm{L}$, hemoglobin $136 \mathrm{~g} / \mathrm{L}$, and platelets 250x10 $/ \mathrm{L}$. ESR was $87 \mathrm{~mm} / \mathrm{h}$ and CRP was $327.4 \mathrm{mg} / \mathrm{L}$. A review of prior serology showed positive ANA, anti-dsDNA antibodies and SSA/Ro antibodies.

Cerebrospinal fluid analysis proved unremarkable with glucose at $3.4 \mathrm{mmol} / \mathrm{L}$ (serum glucose $4.5 \mathrm{mmol} / \mathrm{L}$ ), protein at $275 \mathrm{mg} / \mathrm{L}$, and 4 nucleated cells $/ \mathrm{mm}^{3}$. CSF cytology was negative. All CSF investigations for infectious etiologies were negative: viral PCR, viral culture, gram stain, bacterial culture, fungal culture, cryptococcal antigen, acid fast bacilli, VDRL, and toxoplasma IgM. Serology for histoplasma antibody, cryptococcal antigen, as well as blood cultures and urine cultures for bacteria and fungi were negative.

An EEG demonstrated diffuse slowing (delta) but no lateralizing findings or epileptiform activity.

The original CT scan demonstrated a single left frontal lesion with a small circular focus of hyperdensity surrounded by edema. Magnetic Resonance imaging post gadolinium demonstrated multiple enhancing small lesions in the right frontal lobe, left frontal lobe, left internal capsule and left caudate nucleus (see Figure 1).

\section{DR. TARTAGLia}

The multiple enhancing lesions on MRI could be infectious, inflammatory, or neoplastic either primary or secondary. The negative viral, bacterial, fungal and parasitic cultures do not

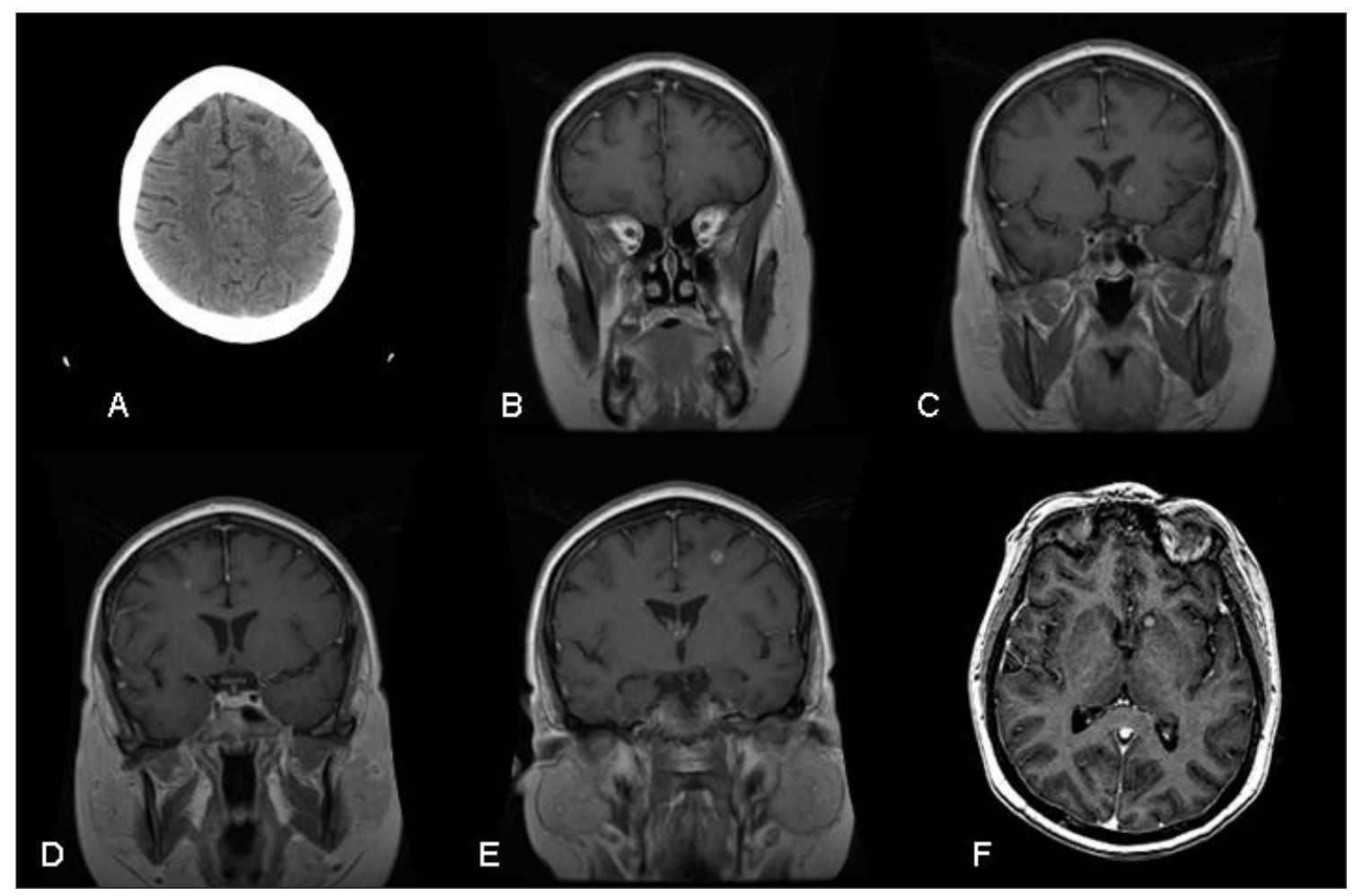

Figure 1: A. Axial CT demonstrates a small left frontal hyperdense lesion with surrounding edema. B-E. (coronal) and F. (axial) T1-weighted MRI with gadolinium demonstrates multiple supratentorial enhancing lesions - left and right frontal lobes, left caudate nucleus, and left internal capsule. 
completely rule out infections as abscesses can give falsenegative results. I would proceed to a brain biopsy.

\section{DR. STECKLEY}

She continued to be febrile with respiratory difficulty and four days later she required intubation for hypoxia. A CT scan of the thorax demonstrated mild ground-glass infiltrates. While awaiting laboratory results, she was treated empirically with antibiotics and antifungal agents, as well as with pyrimethamine, azithromycin, and folinic acid for possible CNS toxoplasmosis. Viral PCR, fungal cultures and AFB testing was negative on samples obtained from bronchioalveolar lavage. A brain biopsy was not offered by the neurosurgical service because of the small size of the lesions and on account of her fragile medical state. Treatment for CNS toxoplasmosis was continued. Her acidosis worsened and she developed renal failure. Despite dialysis, she continued to deteriorate. Life support was withdrawn and she died 11 days after her admission. Consent for a full autopsy was granted.

\section{Dr. TARTaglia}

The lack of response to empiric antimicrobial agents, including treatment for CNS toxoplasmosis, as well as the progressive decline points toward a non-infectious etiology. I would be most concerned for a multifocal malignant process.

\section{DR. REDDY}

The brain was symmetrical and not swollen. On coronal sections, three well-circumscribed firm yellow/tan nodules were found in the cerebral hemispheres each measuring approximately $1 \mathrm{~cm}$ in diameter. The lesions were located in the left internal capsule, the high left parasagittal white matter, and the right periventricular white matter, near the head of the caudate (Figure 2A,B).

Microscopic examination of nodules revealed collections of discohesive atypical mononuclear cells with showed necrotic cores and surrounding edema (Figure 2C). The infiltrates were largely angiocentric, but more diffusely infiltrative elements were also apparent (Figure 2D). Reticulin stains revealed redundant perivascular wrappings which invested layers of angiocentric infiltrates to demonstrate angioinvasion by the lesional cells (Figure 2E).

The atypical cells displayed irregularly shaped nuclei, densely clumped chromatin, and scant cytoplasm. Mitoses were readily found. They expressed abundant CD20 (Figure 2F) and faint CD79, both of which are markers for B-cell lineage. T-cell markers were negative and there was no expression of EBV antigens.

Further focal infiltrates were also found in the meninges, anterior pituitary gland, and choroid plexus.

\section{Final Neuropathological Diagnosis}

High grade large B-cell lymphoma (involving left and right frontal white matter, left internal capsule, pituitary gland, choroid plexus and meninges). General autopsy demonstrated further extensive lymphomatous disease involving periaortic lymph nodes, myocardium, liver, spleen, kidney, and adrenal glands.
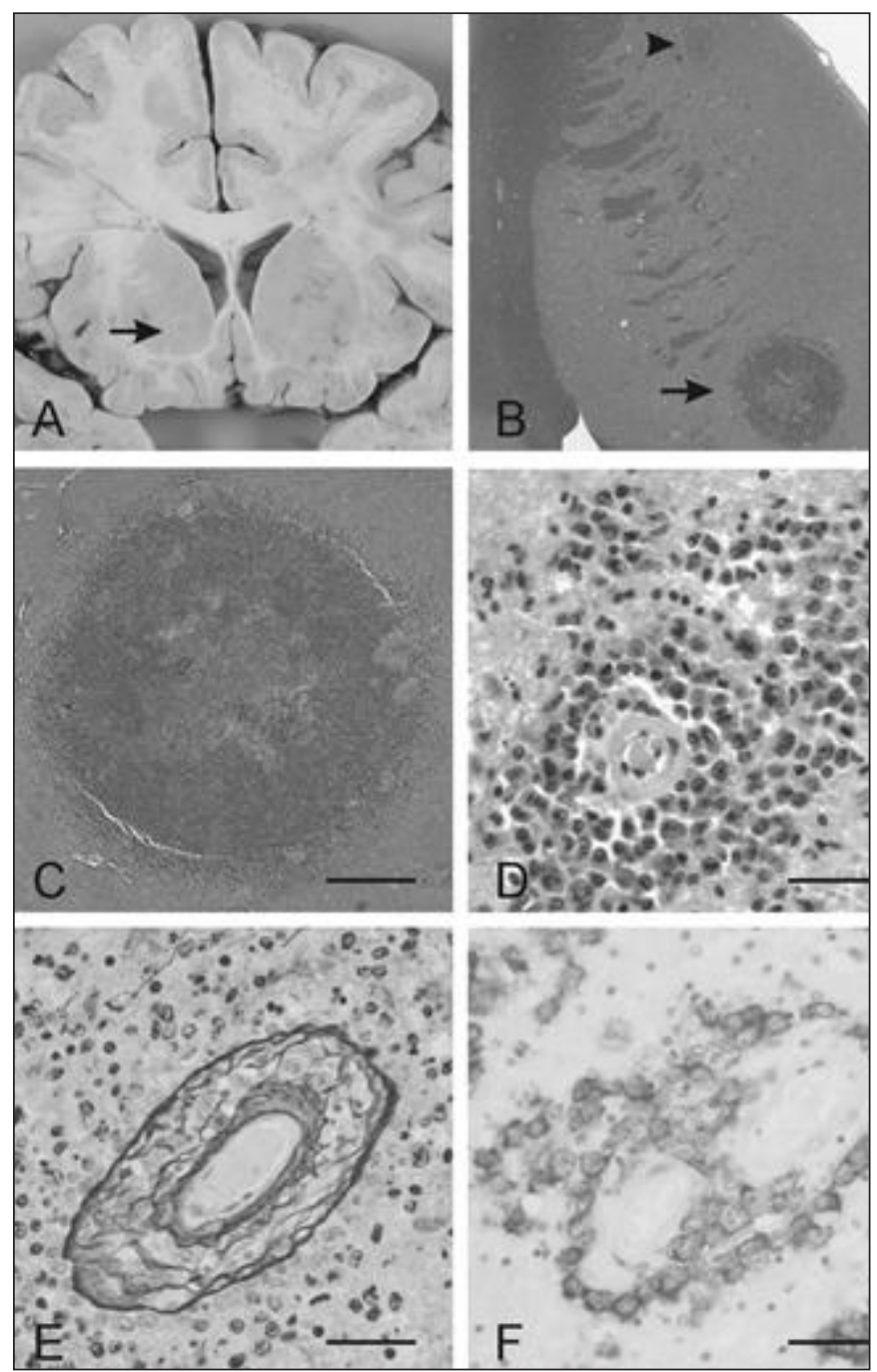

Figure 2: A. Coronal section of cerebrum after fixation. A $5 \mathrm{~mm}$ diameter lesion (arrow) is present within the left basal ganglia and anterior limb of internal capsule corresponding to the lesion identified in Figure 1C. $B$. The same lesion (arrow) is shown in a whole mount histological section as well as a smaller focus (arrowhead) in the caudate head $(H \& E)$. C. The larger lymphomatous deposits had partly necrotic cores. At the periphery, an angiocentric tendency gave way to shallow single cell infiltration into adjacent parenchyma $(H \& E$, bar $=1 \mathrm{~mm}) . D$. The discohesive neoplastic cells display prominent angiocentricity, pleomorphism, mitotic activity and dense chromatin patterns $(H \& E$, bar $=50 \mathrm{um}$ ). E. Angioinvasion by lymphomatous cells (reticulin, bar $=50$ um). F. The lesional cells express the B-cell lineage antigen, CD2O (antiCD20 immunoperoxidase, bar $=50 \mathrm{um}$ )

\section{DisCUSSION}

\section{Neurological involvement in SLE}

Systemic lupus erythematosus is a common multisystem immune-mediated disorder with a prevalence of 6-50 per 100,000 . Current opinion is that $50 \%$ of SLE patients will have neuropsychiatric manifestations. ${ }^{1}$ CNS lupus may cause aseptic meningitis, vasculopathy and cerebrovascular disease, 
demyelination, headache, movement disorders (chorea, myoclonus, parkinsonism), seizures, acute confusional state, anxiety, psychosis, mood disorder, cognitive dysfunction or myelopathy. Various peripheral manifestations may also occur such as peripheral neuropathy and myasthenia gravis. ${ }^{2}$ Other causes of neurologic dysfunction in SLE relate to immune suppression and include opportunistic infections as well as malignancies such as lymphoma.

\section{Brief summary of case}

In the present case, multiple CNS lesions in a patient with SLE presented a diagnostic challenge. Our primary consideration was an infectious etiology and thus empiric treatment was initiated. At autopsy, multiple CNS lesions were found to be due to CNS invasion by a systemic large B cell lymphoma, which had also infiltrated the heart, lungs, liver, adrenals, and spleen.

\section{SLE and increased risk of lymphoma}

There is an association of lymphoma with SLE and other rheumatologic diseases. One study showed an increased incidence ratio of lymphoma in SLE patients of 3.6 (95\% CI 2.64.9 ), with a female preponderance and commonly aggressive histological subtypes, such as diffuse large B cell variants. ${ }^{3}$ A recent meta-analysis confirmed a moderate risk for lymphoma in SLE (random effects SIR 7.4; 95\% CI 3.3-17.0). ${ }^{4}$ The elevated risk of lymphoma is presumed to be due to longstanding inflammatory activity and B cell activation.

\section{Immuno-suppression and lymphoma risk}

It is well known that immunosuppression increases the risk of lymphoma. ${ }^{5}$ An increased risk of lymphoma is seen with azathioprine treatment in rheumatoid arthritis, ${ }^{5}$ organ transplantation, ${ }^{6}$ and inflammatory bowel disease where there is a 4-fold increase in risk. ${ }^{7}$ Azathioprine may have been a contributing factor to the development of lymphoma in our case. Alternatively, azathioprine treatment may be a confounding marker of severe immune-mediated disease, which by itself may increase the risk of lymphoma. Although there may be a small increased risk of lymphoma with azathioprine therapy, it is counterbalanced in long term medical management by a reduction in the inflammatory disease burden.

\section{REFERENCES}

1. Joseph FG, Lammie GA, Scolding NJ. CNS lupus. Neurology. 2007;69:644-54.

2. The American College of Rheumatology nomenclature and case definitions for neuropsychiatric lupus syndromes. Arthritis Rheum. 1999;42:599-608.

3. Bernatsky S, Ramsey-Goldman R, Rajan R, Boivon J-F, Joseph L, Lachance $\mathrm{S}$, et al. Non-Hodgkin's lymphoma in systemic lupus erythematosus. Ann Rheum Dis. 2005;64:1507-9.

4. Zintzaras E, Voulgarelis M, Moutsopoulos HM. The risk of lymphoma development in autoimmune diseases. Arch Intern Med. 2005;165:2337-44.

5. Asten P, Barrett J, Symmons D. Risk of developing certain malignancies is related to duration of immunosuppressive drug exposure in patients with rheumatic diseases. J Rheumatol. 1999;26:1705-14.

6. Smith JL, Wilkinson AH, Hunsicker LG, Tobacman J, Kapelanski DP, Johnson M, et al. Increased frequency of post transplant lymphomas in patients treated with cyclosporine, azathioprine and prednisone. Transplant Proc. 1989;21(1 Pt 3):3199-200.

7. Kandiel A, Fraser AG, Korelitz BI, Brensinger C, Lewis JD. Increased risk of lymphoma among inflammatory bowel disease patients treated with azathioprine and 6-mercaptopurine. Gut. 2005;54:1121-5. 